\title{
Sensitivity of TEM data on lightspeed to camera-length's voltage variation
}

\author{
David Osborn $^{1}$, Tianna McBroom ${ }^{1}$ and P. Fraundorf ${ }^{1}$ \\ ${ }^{1}$ Physics \& Astronomy Dept. \& Center for Nanoscience, U. Missouri-Saint Louis, MO 63121 USA
}

As one might imagine, the behavior of electrons which approach or exceed the speed of light in water is likely to be affected by the size of the spacetime constant c (literally the number of meters in a second) commonly called "lightspeed". For instance the free-particle dispersion relation (e.g. temporal-frequency versus wavenumber), plotted as kinetic energy $\mathrm{K}$ versus momentum $\mathrm{p}$ for a given mass, would according to Newton have been a straight line on a log-log plot with slope $\alpha=2$ of the form $\mathrm{K}=\mathrm{p}^{2} /(2 \mathrm{~m})$. For any given value of $\mathrm{p}$, all values of $\mathrm{K}$ would have been possible.

At high speeds, we now know that this line curves over to join the mass-independent "photon line" $\mathrm{K}=$ pc. This has a slope of 1 instead of 2 on the same log-log plot. That photon-line represents the bottom of the "lightspeed curtain", which requires instead that no objects may be found with K greater than the upper limit of pc.

Fig. 1 shows that common TEM electron energy/momenta lie on the curved part of this plot, which suggests that one can use them to determine the value of spacetime constant $\mathrm{c}$ from their behavior alone. We propose here a "ratio-method", which involves only dimensionless measurements of the ratio, at two different operating voltages, of three quantities associated with a diffraction ring from the same polycrystalline specimen, namely electron voltage $\mathrm{V}$, ring radius $\mathrm{g}$, and camera length $\mathrm{L}$.

In order to minimize the number of constants needed for this log-log slope measurement, note that the slope $\alpha=(\mathrm{p} / \mathrm{K}) \delta \mathrm{K} / \delta \mathrm{p}=1+1 /\left(1+\mathrm{K} /\left(\mathrm{m}_{\mathrm{e}} \mathrm{c}^{2}\right)\right)$. Since $\mathrm{K}=\mathrm{q}_{\mathrm{e}} \mathrm{V} \propto \mathrm{V}$ and $\mathrm{p}=\mathrm{hL} / \mathrm{g}_{\mathrm{hkl}} \mathrm{d}_{\mathrm{hkl}} \propto \mathrm{L} / \mathrm{g}$, one can approximate $\alpha$ at the halfway point between voltages $\mathrm{V}_{1}$ and $\mathrm{V}_{2}$ from (hkl) diffraction-ring radius $\mathrm{g}$ measurements (like those depicted in Fig. 2$)$ at those voltages using $\alpha \approx\left(1-\mathrm{V}_{2} / \mathrm{V}_{1}\right) /\left(1+\mathrm{V}_{2} / \mathrm{V}_{1}\right) \times$ $\left(\mathrm{g}_{2} / \mathrm{g}_{1}+\mathrm{L}_{2} / \mathrm{L}_{1}\right) /\left(\mathrm{g}_{2} / \mathrm{g}_{1}-\mathrm{L}_{2} / \mathrm{L}_{1}\right)$, allowing one to determine $\left(\mathrm{m}_{\mathrm{e}} / \mathrm{q}_{\mathrm{e}}\right) \mathrm{c}^{2}=\mathrm{V}(\alpha-1) /(2-\alpha)$ as well as $\alpha$ from only $\mathrm{g}$, $\mathrm{L}$ ratios and $\mathrm{V}$.

Fig. 3 shows the relationship between the measured slope $\alpha$ and lightspeed c for various voltages, assuming that the electron charge/mass ratio $\left(\mathrm{q}_{\mathrm{e}} / \mathrm{m}_{\mathrm{e}}\right)$ is known. The inferred value of lightspeed $\mathrm{c}$ is highly sensitive to errors in the camera-length $\left(\mathrm{L}_{2} / \mathrm{L}_{1}\right)$ ratio. For example, "book values" for L-ratios between 300, 250, and $200 \mathrm{keV}$ in our EM430ST leads one to c-values which are all over the map for a set of polycrystal-aluminum film (111) diffraction-ring radius measurements.

By comparison the red and cyan spot triplets on the plot show values for c inferred from two independent pairs of measurements from the same patterns, using not "book values" but instead L-ratios optimized to minimize the spread associated with both datasets. In this case the spread in predictions for c settled down to a standard deviation about the mean of about $20 \%$.

Camera-length ratios can in principle be determined empirically by electron "ray" optics in a way which is also independent of fundamental constants. As a first step to this end we recommend recording the image of a large objective aperture as an internal standard in each diffraction pattern (as also shown in 
Fig. 2). In the thin objective-lens approximation, the diameter of this aperture will be proportional to camera length so that the camera-length ratio can be estimated from the aperture-diameter ratio.

In general, however, the objective aperture size is strongly affected by objective-lens strength as well as by camera-length changes. We suspect that knowledge of objective-lens current ratios, coupled with a thick lens geometry factor, will allow one to use the objective-aperture size ratio as a measure of camera-length ratio nonetheless. Experts in lens design for individual microscopes might be able to help in that context.

The sensitivity of this measurement to knowledge of camera-length ratios between different voltages suggests that it may also serve as a metrology check, for groups planning to use their microscopes for comparison of measurements at more than one voltage. In that context, we plan to set up a database for users to share and discuss measurements from various microscopes at sites.google.com/site/electrondetectives/.

\section{References:}

[1] Tavish L. E. Hill and P. Fraundorf (2015) "A Spacetime-Constant Experiment Using Electrons", American Association of Physics Teachers 2015 Summer Meeting Program Book, page 57
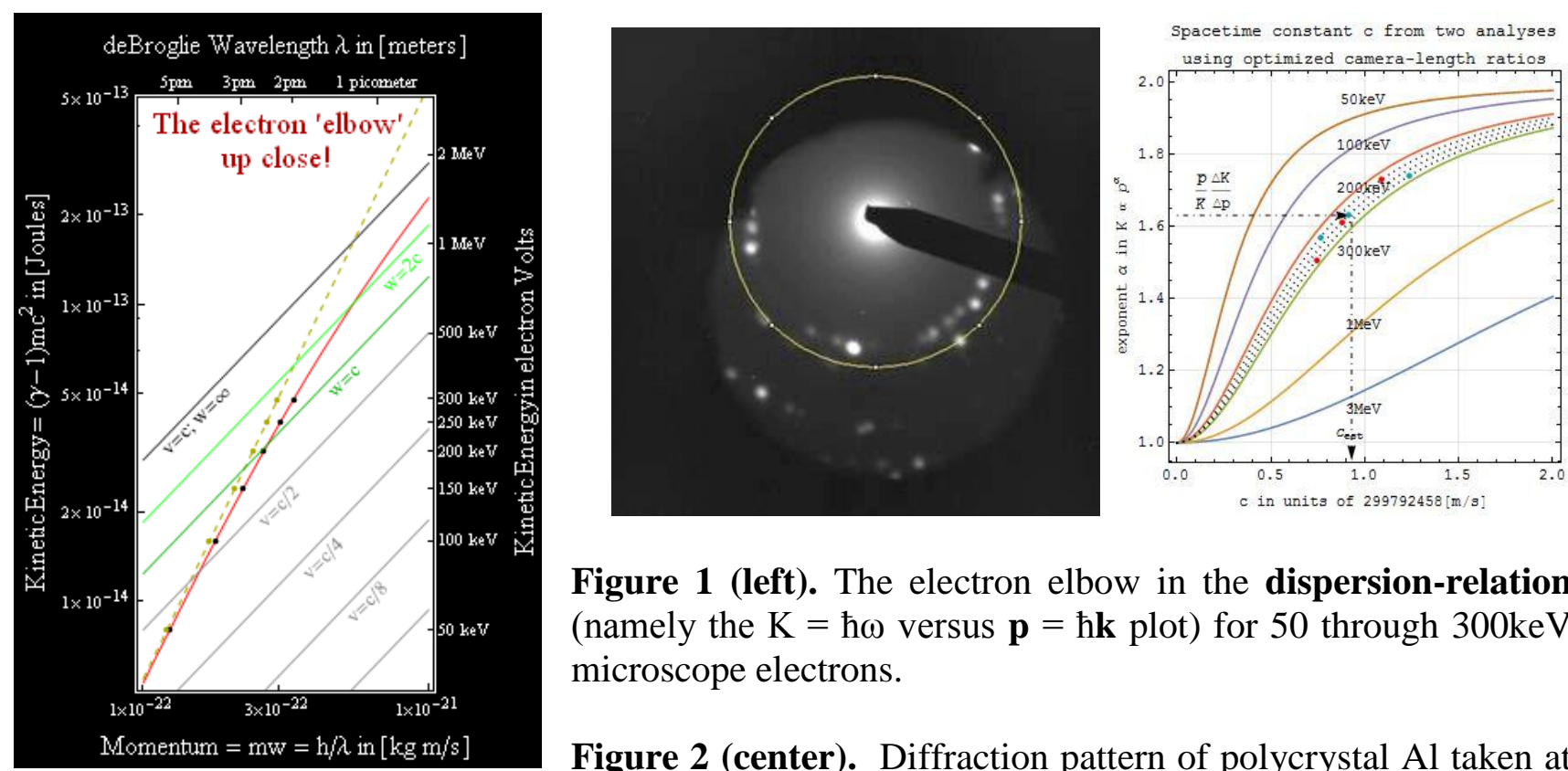

Figure 1 (left). The electron elbow in the dispersion-relation (namely the $\mathrm{K}=\hbar \omega$ versus $\mathbf{p}=\hbar \mathbf{k}$ plot) for 50 through $300 \mathrm{keV}$ microscope electrons.

Figure 2 (center). Diffraction pattern of polycrystal Al taken at one of 3 voltages, with large objective aperture and a (200) ring circle from ImageJ for estimating ring radius.

Figure 3 (right). The red and cyan dots denote the triplet of values for slope and lightspeed obtained by two observers making measurements from the same four diffraction negatives, and converted to slopes using a set of camera-length ratios which were optimized to give us the best agreement. 